\title{
Relations entre la dégradabilité de l'azote des aliments concentrés dans le rumen et sa digestibilité dans l'intestin, et certains critères biochimiques
}

\author{
J Aufrère, B Michalet-Doreau, D Graviou \\ INRA, Station de Recherches sur la Nutrition des Herbivores, \\ Unité de la Valeur Alimentaire, INRA, Theix, 63122 Ceyrat, France
}

Summary - Estimation of intestinal nitrogen digestibility was related to acid detergent-insoluble nitrogen (ADIN) or pepsin-insoluble nitrogen (IP). Feedstuffs were then classified according to their in sacco nitrogen degradability in the rumen and $A D I N$ or IP.

Introduction - Dans le système français d'estimation de la valeur azotée des aliments (protéines digestibles dans l'intestin: PDI) (Vérité et al, 1987), la dégradabilité de l'azote dans le rumen ou dégradabilité théorique de l'azote (DT) est mesurée par la technique des sachets de nylon. La digestibilité réelle dans l'intestin (dr) est calculée en prenant en compte les teneurs en matières azotées non digestibles (MAND) diminuées des composantes non alimentaires (microbiennes et endogènes) des fèces. L'objet de ce travail est d'établir un classement relatif des aliments en fonction de la digestion de leur azote dans le rumen ou l'intestin, à partir de critères biochimiques: azote de la fraction ADF (ADIN) et azote insoluble dans la pepsine (IP).

Matériel et Méthodes - L'étude a porté sur 57 échantillons d'aliment correspondant à 24 aliments concentrés simples différents. Pour chaque échantillon on a déterminé la teneur en azote total (Nt) par la méthode de Kjeldhal, la DT calculée à partir de la cinétique de dégradation in sacco dans le rumen en admettant un taux de sortie des particules du rumen de $6 \% / \mathrm{h}$ (Vérité et al, 1987), les teneurs en ADIN et IP (Goering et al, 1972).
Résultats et Discussion - Pour les $\mathbf{5 7}$ aliments, il existe des relations étroites entre la dr retenue pour les Tables (Vérité et al, 1987) et, soit l'ADIN (écart type résiduel $S r=0,05$ ), soit I'IP ( $S r=0,06$ ) (fig 1) si on excepte les graines protéagineuses (féverole, lupin et colza) pour lesquelles la dr est faible $(\approx 0,60)$ et vraisemblablement sous-estimée. En effet, pour les aliments dont l'azote est très dégradé, la dr calculée diminue très rapidement dès qu'on surestime la teneur en MAND. L'ADIN et I'IP sont donc de bons indicateurs de la dr. En revanche, la DT n'est que faiblement liée à ces paramètres $(r=-0,35$ pour l'ADIN et - 0,31 pour l'IP).

Toutefois, l'examen de la fig 2 permet d'identifier différentes populations d'aljments en fonction de leur comportement dans le rumen et dans l'intestin : 1) les aliments dont la DT est supérieure à $70 \%$ (les oléagineux, les protéagineux, les céréales sauf le maïs, certains tourteaux) et dont les proportions en ADIN ou IP sont faibles (en moyenne respectivement de 2,5 et $12 \% \mathrm{Nt}$ ). L'azote de ces aliments arrive en faible proportion au duodénum mais présente une dr élevée; 2) les aliments dont la DT est comprise entre 55 et 


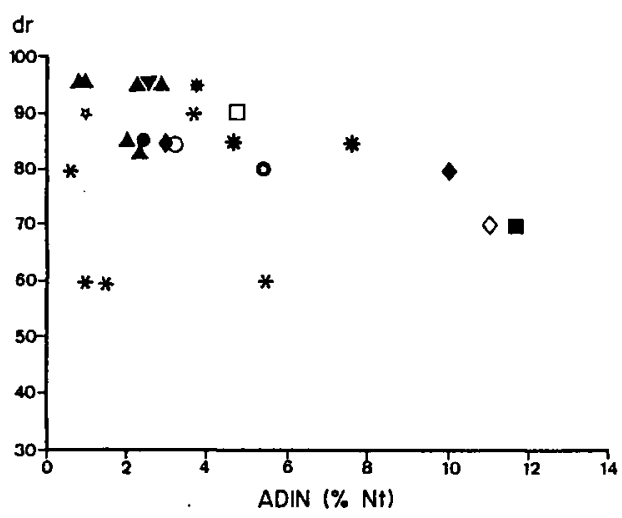

Fig 1. Liaison entre la dr et l'ADIN.

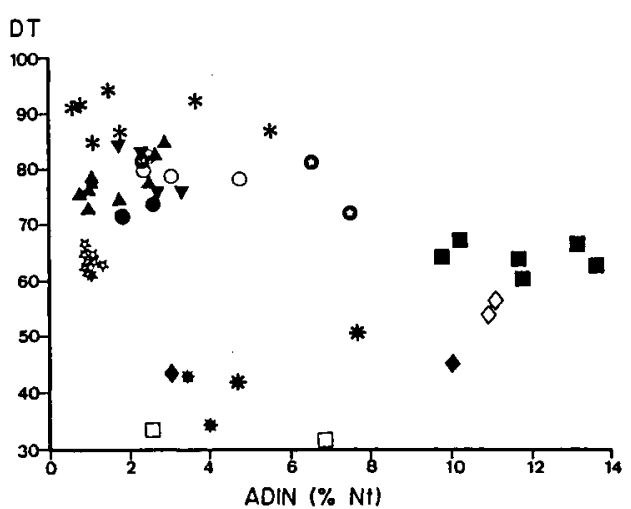

Fig 2. Liaison entre la DT et l'ADIN.

(* tourteaux (Tx) soja, $\$$ Tx colza, $O T x$ tourneseol, $-T x$ arachide, $\square T x$ tannés, $\Delta$ céréales (sauf maïs), $\nabla$ sous-produit (SP) céréales, * maïs, * SP maïs, * graines légumineuses, $\mathbf{a}$ luzerne déshydratée, $O$ pulpe betterave, $O$ SP animaux).

$70 \%$, et dont l'azote est donc moyennement dégradés dans le rumen, pour lesquels les teneurs en ADIN ou IP sont, soit faibles (en moyenne respectivement 0,9 et $7,1 \% \mathrm{Nt}$ ) pour le tourteau de soja, soit élevées (12 et $25 \% \mathrm{Nt}$ ) pour la luzerne ou la pulpe de betterave déshydratées. L'azote de ces aliments arrivant au duodénum est donc, soit très digestible, soit beaucoup moins et, dans ce dernier cas, vraisemblablement par suite de réactions de Maillard formées lors de leur déshydratation. C'est sans doute aussi le chauffage qu'ils ont subi qui explique leur DT moyenne; 3) enfin des aliments dont la DT est faible (30 $<$ DT < 50\%) et qui ont des teneurs en ADIN ou IP variables $(2<$ ADIN $<10 \% ~ N t ;$ $8<\mathrm{IP}<26 \% \mathrm{Nt}$ ). Ce dernier groupe est constitué du maïs et ses co-produits, de sous-produits animaux (farine de viande et de poisson) et des tourteaux tannés. Ces aliments, peu dégradés dans le rumen, ont une dr variable dans l'intestin $(60<\mathrm{dr}<$ 95\%).

En conclusion, en combinant la DT et I'ADIN ou I'IP, il est possible de classer l'azote des aliments en fonction de son aptitude non seulement à être dégradé dans le rumen, mais aussi à être digéré dans l'intestin grêle.

Goering $\mathrm{HK}$, Gordon $\mathrm{CH}$, Hemken RW, Waldo DR, van Soest PJ, Smith LW (1972) J Dairy Sci $55,1275-1280$

Vérité R, Michalet-Doreau B, Chapoutot P, Peyraud $J L$, Poncet $C$ (1987) Bull Tech CRZV Theix, INRA 70, 19-34 